\title{
Effect of shear force on the separation of double stranded DNA
}

\author{
Rakesh Kumar Mishra, Garima Mishra, M. S. Li ${ }^{1}$ and Sanjay Kumar \\ Department of Physics, Banaras Hindu University, Varanasi 221 005, India \\ ${ }^{1}$ Institute of Physics, Polish Academy of Sciences, Al. Lotnikow 32/46, 02-668 Warsaw, Poland
}

\begin{abstract}
Using the Langevin Dynamics simulation, we have studied the effects of the shear force on the rupture of short double stranded DNA at different temperatures. We show that the rupture force increases linearly with the chain length and approaches to the asymptotic value in accordance with the experiment. The qualitative nature of these curves almost remains same for different temperatures but with a shift in the force. We observe three different regimes in the extension of covalent bonds (back bone) under the shear force.
\end{abstract}

PACS numbers: 87.15.A-,64.70.qd,05.90.+m,82.37.Rs

Inter- and intra- molecular forces are key to the stability of DNA and biological processes e.g. transcription, replication, slippage etc. [1, 2]. Up to now, understanding of these forces was possible through the indirect physical and thermodynamical measurements like crystallography, light scattering, nuclear magnetic resonance spectroscopy etc. [3]. Single molecule force spectroscopy (SMFS) experiments have directly measured these forces and provided unexpected insights into the strength of the forces driving these biological processes as well as determined various interactions responsible for the mechanical stability of DNA structures [4 7]. With the increasing number of experiments and insights gathered so far, it has become clear that the measurement of molecular interactions not only depends on the magnitude of the applied force, but also depends how and where the force was applied 7 [15].

A major concern is now to understand whether all these interactions contribute at the same moment or they have different life times. In order to understand this, a force has been applied perpendicular to the helix direction (DNA unzipping) and along the helix direction (rupture and slippage) as shown in Fig. 1 [7-16]. In case of unzipping of double stranded DNA (dsDNA), the critical force is found to be independent of the length of DNA and the loading rate [8, 9]. This may be understood theoretically that at the centre point of fork (Fig. 1b), the applied force only breaks a base pair at a time and hence it remains independent of the loading rate and length. However, when a force (up to $65 \mathrm{pN}$ ) is applied along the helix direction (shear force), the length of the dsDNA increases and the force-extension $(f-x)$ curve can be described by the worm like chain (WLC) model [17]. In the high force regime $(>65 \mathrm{pN})$, the dsDNA can be overstretched about 1.7 times of the Bform contour length and a phase transition occurs from the B-form to a stretched or S-form 18 20]. Recently, van Mameren et al. have studied DNA stretching with or without DNA binding ligands and demonstrated that overstretching comprises a gradual conversion from dsDNA to ssDNA and it should be interpreted in terms of force induced DNA melting [21].

For a short dsDNA, if the applied shear force increases,

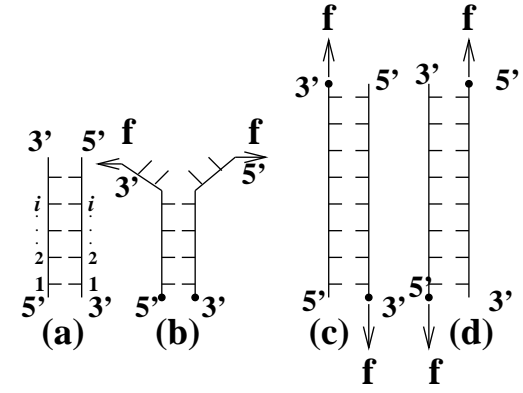

FIG. 1: Schematic representation of dsDNA: (a) dsDNA in zipped form; (b) Unzipping of dsDNA by the force $(f)$ applied at one end $\left(5^{\prime}-3^{\prime}\right)$; (c and d) Shear force along the chain applied at the opposite ends $\left(3^{\prime}-3^{\prime}\right.$ or $\left.5^{\prime}-5^{\prime}\right)$ of the dsDNA.

the dsDNA separates into two single strands at some critical force. This phenomenon has been identified as rupture [6, 10]. The unbinding force strongly depends on the pulling end and is much larger than the unzipping force [6, 10, 14, 22]. Neher and Gerland studied the dynamics of dissociation of the two strands and found the expression for the critical force [23]. Expressing the bond energy and the base pairing energy in the form of harmonic oscillators in the ladder model of dsDNA of length $L$, de Gennes [24] proposed the maximum force required for the rupture

$$
f_{c}=2 f_{1}\left(\chi^{-1} \tanh \left(\chi \frac{L}{2}\right)+1\right) .
$$

Here, $f_{1}$ is the force required to separate a single base pair, which is same for the homo-sequence and $\chi^{-1}=\sqrt{Q / 2 R}$ is the de Gennes characteristic length over which differential force is distributed. Here, Q and $\mathrm{R}$ are the spring constants, characteristic of stretching of backbone and hydrogen bonds, respectively.

Recently, Danilowicz et al. [13] systematically studied the DNA rupture by varying the length of dsDNA. The critical shear force is found to increase linearly up to a certain length and approaches the asymptotic value $(\approx 62 \mathrm{pN})$, which is in good agreement with the de Gennes prediction. It was argued that the covalent bonds (backbone) and the hydrogen bonds involved in the base 
pairing will be stretched under the applied force. The differential force will approach to zero at the length $\chi^{-1}$, if one moves in from the either side. However, no experimental effort has been made to study the effect of shearing force on the stretching of covalent bonds and hydrogen bonds in side the characteristic length $\chi^{-1}$. Moreover, in the description of de Gennes model [24] or subsequently improved model by Chakrabarti and Nelson [25], effect of thermal fluctuation has been ignored, whereas all the rupture experiments were generally performed at finite temperature. The aim of this manuscript is to study the effect of temperature $(T)$ on the rupture and consequences of differential force on the distribution of extension in bond lengths and hydrogen bonds near the rupture.

We use Langevin Dynamics (LD)simulation to investigate mechanical and physical properties related to the rupture of DNA [26, 27, 29, 30]. Since, rupture time is of the order of milliseconds to seconds, an atomistic simulation of longer chain in the solvent is computationally difficult 31, 32]. We have used a coarse-grained model [7, 29, 30, 33] of the flexible polymer chain to model a DNA, which allows us to study a larger system size and events of a longer time scale. A chain in the model consists of bead units connected by effective bonds characterized by the stiff springs. Each effective bond represents several chemical bonds (e.g. sugar phosphate etc. ) along the chain backbone. The energy of the model system is given by

$$
\begin{aligned}
& E=\sum_{l=1}^{2} \sum_{j=1}^{N} k\left(u_{j+1, j}^{(l)}-d_{0}\right)^{2}+\sum_{l=1}^{2} \sum_{i=1}^{N-2} \sum_{j>i+1}^{N} 4\left(\frac{C}{u_{i, j}^{(l)}}\right) \\
& +\sum_{i=1}^{N} \sum_{j=1}^{N} 4\left(\frac{C}{\left.\left(\mid \vec{u}_{i}^{(1)}-\vec{u}_{j}^{(2)}\right)\right|^{12}}-\frac{A}{\left(\left|\vec{u}_{i}^{(1)}-\vec{u}_{j}^{(2)}\right|\right)^{6}} \delta_{i j}\right),(2)
\end{aligned}
$$

where $N$ is the number of beads in each strand. $\vec{u}_{i}^{(l)}$ represents the position of $i^{t h}$ bead on $l^{\text {th }}$ strand. In present case, $l=1(2)$ corresponds to first (complimentary) strand of dsDNA. The distance between intra strand beads, $u_{i, j}^{(l)}$, is defined as $\left|\vec{u}_{i}^{(l)}-\vec{u}_{j}^{(l)}\right|$. The harmonic (first) term with spring constant $k(=100)$ couples the adjacent beads along the two strands. Second term takes care of excluded volume effect i.e. two beads can not occupy the same space [34]. The third term, described by Lennard-Jones (LJ) potential, takes care of the mutual interaction between two strands. The first term of LJ potential (same as second term of Eq.2) will not allow the overlap of two strands. Here, we set $C=1$ and $A=1$. The second term of LJ potential corresponds to the base pairing between two strands. The base pairing interaction is restricted to the native contacts $\left(\delta_{i j}=1\right)$ only i.e. $i^{\text {th }}$ base of $1^{\text {st }}$ strand forms pair with the $i^{\text {th }}$ base of $2^{\text {nd }}$ strand only as shown in Fig. 1a. This is similar to the Go model [28]. The parameter $d_{0}(=1.12)$ corresponds to the equilibrium distance in the harmonic potential, which is close to the equilibrium position of
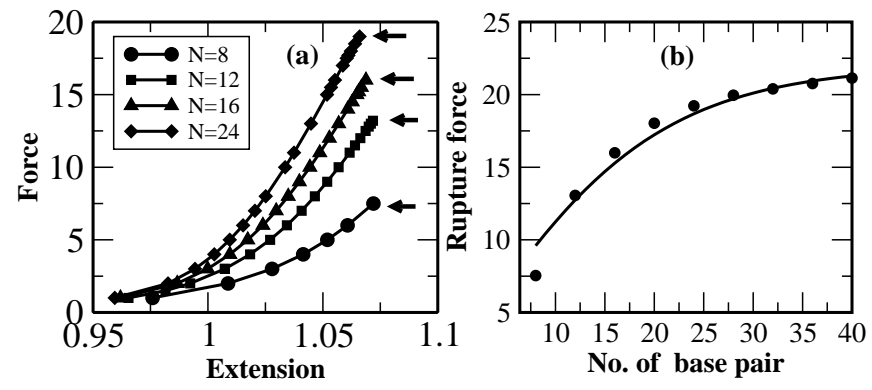

FIG. 2: (a) Force vs extension curves for different chain lengths Arrows indicate the maximum force, where the number of contacts approaches to zero. For the sake of comparison, we have normalized the extension by its contour length $($ at $\mathrm{f}=0)$. (b) Figure shows the variation of rupture force with length. The solid line corresponds to a fit of Eq. 1. Solid circles represent the value obtained through the simulation.

the average LJ potential. In Eq. 2, we use dimensionless distances and energy parameters. The major advantage of this model is that the ground state energy of the system is known [28]. The equation of motion is obtained from the following Langevin equation [26, 27, 30]

$$
m \frac{d^{2} r}{d t^{2}}=-\zeta \frac{d r}{d t}+F_{c}+\Gamma
$$

where $m$ and $\zeta$ are the mass of a bead and the friction coefficient, respectively. Here, $F_{c}$ is defined as $-\frac{d E}{d r}$ and the random force $\Gamma$ is a white noise [27], i.e., $<\Gamma(t) \Gamma\left(t^{\prime}\right)>=2 \zeta T \delta\left(t-t^{\prime}\right)$. The choice of this dynamics keeps $T$ constant throughout the simulation for a given $f$. The equation of motion is integrated by using the $6^{\text {th }}$ order predictor-corrector algorithm with time step $\delta t=0.025$ [27]. The results are averaged over many trajectories. The equilibration has been checked by monitoring the stability of data against at least ten times longer run. We have used $2 \times 10^{9}$ time steps out of which first $5 \times 10^{8}$ steps have not been taken in the averaging.

In the constant force ensemble, we add an energy $-\vec{f} . \vec{x}$ to the total energy of the system given by Eq. 2. We calculate the reaction coordinate $x$ (extension) for different values of $f$. The $f-x$ curves (Fig. 2a) show the entropic response at low forces and remain qualitatively similar to the one seen in experiments [6, 10, 11, 15]. We identify the rupture force as a maximum force, where the number of intact base pairs suddenly goes to zero. In Fig. 2b, we show the rupture force as a function of the chain length at low temperature. It is evident from this plot that the rupture force approaches to an asymptotic value for the chain length greater than 20, which is in accordance with the experiment [13].

We expand the LJ potential given in Eq.2 around its equilibrium value. The coefficient of second (harmonic) term of its expansion corresponds to the elastic constant of the base-pairing. The de Gennes characteristic length 

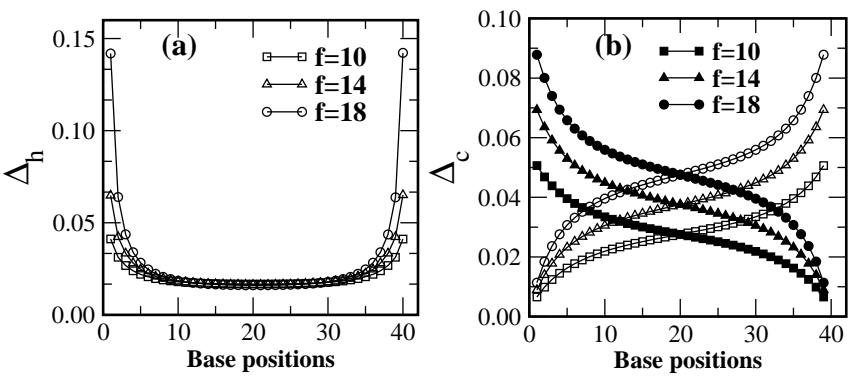

FIG. 3: (a) Variation of extension in hydrogen bond length $\left(\Delta_{h}\right)$ along the chain at three different forces. (b) Figure shows the variation in extension of covalent bond length $\left(\Delta_{c}\right)$ along the chain. Here, open and solid symbols correspond to one strand and its complementary strand, respectively.

[24] for the present model is estimated to be $\approx 10$. Substituting the value of $f_{1}(=1)$ and the above mentioned value of $\chi^{-1}$ in Eq. 1, we obtained the value of $f_{c}$ for a given length of dsDNA, which is shown by solid line in Fig $2 b$. One can notice a nice agreement between the simulation and the value predicted by Eq. 1 [24].

One of the important findings of the present simulation is the distribution of stretching of hydrogen bonds $\left(\Delta_{h}\right)$ and extension in the covalent bonds $\left(\Delta_{c}\right)$ for a wide range of force below the rupture, which are experimentally difficult to obtain. In Fig. 3a, we depict the variation of $\Delta_{h}$ with base position for the chain of length 40. From this plot, one can observe that the hydrogen bonds at extreme ends (up to $\approx 10$ bases) get stretched, whereas bases in the middle (above the de Gennes length $\approx 10 \sim 30$ ) remain same indicating that the differential shear force approaches to zero in this region. In Fig. 3b, we show the variation of $\Delta_{c}$ with the base position. All curves have three distinctively different regions. One can observe that bonds near the pulling end (say 5'-end) get stretched more and decreases gradually. However, when one approaches the other end (i.e. 3'-end), there is a change in the slope and the extension is quite less compare to the middle one. It should be noted that 3'-end is near to 5'-end of the other chain, where a similar force is also applied. Since, dsDNA is in the zipped state, the applied force at 5'-end of one strand also pulls the other strand along the opposite direction, which causes a relatively slower increase.

In model studies either temperature is set to zero 24] or thermal fluctuation is ignored [25] as a result, the rupture force defined in Eq. 1 is independent of temperature. However, all rupture experiments usually performed at room temperature [10, 13], therefore, it is desirable to understand role of entropy, which may play a significant role at higher $T$. The thermodynamics of force induced melting can be obtained from the following relation [35, 36] :

$$
-f x=\Delta H-T \Delta S
$$

where $H$ is the enthalpy and $\mathrm{S}$ is entropy of the ruptured chains. Setting $x$ equal to unity and replacing the value

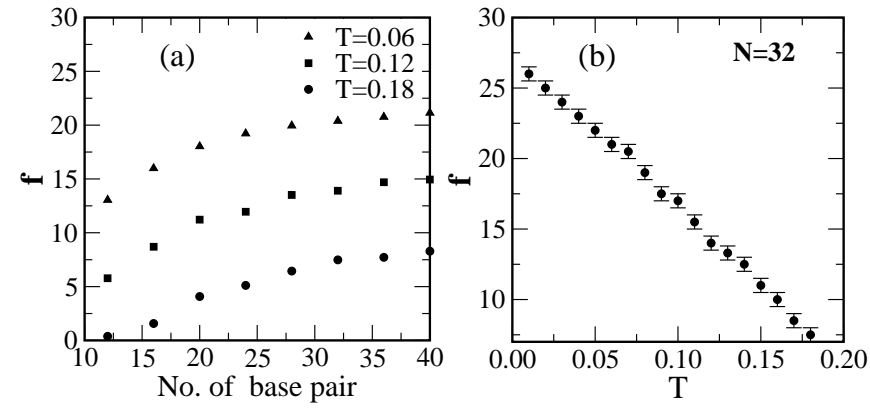

FIG. 4: (a) Variation of the rupture force with chain length at different T. (b) Figure shows the DNA rupture forcetemperature diagram for chain length 32 .

of $\Delta H$ by the value of the rupture force at $T=0$, Eq. 4 can be written as

$$
-f=f_{c}-T \Delta S
$$

where $f_{c}$ is given by Eq. 1 . In the thermodynamic limit, value of $S$ may be analytically estimated [34] or can be obtained from the experiments [37]. However, for the finite chain length, one has to resort on numerical techniques to get the value of $S$. It is possible to study the effect of temperature on the rupture force in the present setup. In Fig. 4a, we show the dependence of the rupture force on the length at different temperatures. The qualitative nature of the curves obtained at different $T$ remain similar to de Gennes plot (Fig. 2b), with a shift showing that the rupture force decreases with $T$. From Fig. 4a, one can notice that for chain length 32 , the rupture force has approached its asymptotic value for all $T$. In Fig. 4b, we depict the force-temperature diagram for the DNA rupture for the chain length 32. A linear dependence on temperature can be noticed, which is in accordance with Eq. 5. Usually rupture experiments are performed well below the melting temperature, which correspond that the DNA is in the zipped state. Therefore, in Fig. 4b, maximum temperature has been set slightly below the melting temperature i.e. $T_{m}=0.23$ at $f=0$.

In this paper, we have studied the effect of shear force and temperature on the rupture of dsDNA. We show that the shear force increases linearly with the length of the DNA and then approaches to the asymptotic value. In the lattice model, the bond length is constant (stiff), therefore, covalent bonds and hydrogen bonds will not be stretched. As a result, the rupture force increases linearly with length 36] in the lattice model or in the models, where bond length is considered as a constant. Our simulations confirm that, one will not gain strength by increasing a larger pair sequence as predicted by de Gennes 24. Interestingly, recent experiment also supports it [13]. The distribution of extension in hydrogen bonds and covalent bonds for different forces are shown in Fig. 3, where Fig. 3a clearly shows that the increase in the extension of the hydrogen bonds is limited up to the de Gennes length, which is consistent with the strain 
profile obtained by Chakrabarti and Nelson 25]. Above this length, differential shear force approaches to zero, as a result there is no extension in the hydrogen bonds. We also find that the qualitative nature of dependence of rupture force on length remains same for different temperatures with a shift. The rupture force decreases with temperature (Fig. 4b) as predicted by Eq. 5 .

Our study shows that the de Gennes length remains independent of the applied force. The most surprising finding of the present simulation is revealed from Fig. 3b, which shows variation of extension in the covalent bonds along the chain for three different forces $(f=10,14,18)$. Although, in all these cases, the differential force approaches to zero and there is no relative increase in the bond length above the de Gennes length along the chain (Fig. 3b). However, unlike the hydrogen bonds, there is a net increase in the extension in the covalent bonds, which depends linearly on the applied force. Nuclear magnetic resonance experiment 38] or the atomistic simulation 32] should be able to observe this.

It may be noted that the present simulation is carried out in the reduced unit. It is possible to extract a rough estimate of the rupture force in the real unit. The free energy per base pair (including hydrogen bonding and base stacking) of $\mathrm{G}-\mathrm{C}$ is $-1.4 \mathrm{kcal} / \mathrm{mol}$, where stacking accounts probably half of this amount [39]. Since in A$\mathrm{T}$ base pairing, only two hydrogen bonds are involved, one can take approximately two third value of the G-C free energy. For fixing the temperature scale, one can use DNA melting data where both stacking as well as hydrogen bonding are required. Whereas in rupture, we assume that there are breaking of hydrogen bonds only and hence stacking does not contribute significantly. In Ref. [13] hetero-sequence ( $50 \%$ AT and 50\% GC) chain of different lengths were considered. Therefore, we take approximately $-0.6 \mathrm{kcal} / \mathrm{mol}$ per base of the zipped conformation and equate it with the complete unzipped state. The required force for the rupture is found to be approximately $3.5 \mathrm{pN}$ per base pair, which is close to the one used in Ref. [13]. So if one scales the y-axis of Fig. 2b by $3.5 \mathrm{pN}$ then our results are also in quantitative agreement with the experiment [13].

We thank D. Giri for many helpful discussions on the subject. Financial supports from the DST, CSIR, India and the MSI, Poland (grant No 202-204-234) are gratefully acknowledged.
[1] B. Alberts et al., Molecular Biology of the Cell, (Garland Publishing: New York, 1994).

[2] J. N. Israelachvili, Intermolecular and Surface Forces (Academic Press, London, 1992).

[3] R. M. Wartell and A. S. Benight, Phys. Rep. 126, 67 (1985).

[4] S. B. Smith, L. Finzi and C. Bustamante, Science 258, 1122 (1992).

[5] P. Cluzel et al., Science 271, 792 (1992).

[6] G. U. Lee, L. A. Chrisey and R. J. Colton, Science 266, 771 (1994).

[7] S. Kumar and M. S. Li, Phys. Rep. 486, 1 (2010).

[8] B. Essevaz-Roulet, U. Bockelmann and F. Heslot, PNAS 94, 11935 (1997).

[9] U. Bockelmann, B. Essevaz-Roulet and F. Heslot, Phys. Rev. Lett. 79, 4489 (1997).

[10] T. Strunz et al., PNAS 96, 11277 (1999).

[11] I. Schumakovitch et al., Biophys. J. 82, 517 (2002).

[12] C. Danilowicz et al., Phys. Rev. Lett. 93, 078101 (2004).

[13] K. Hatch et al., Phys. Rev. E 78, 011920 (2008).

[14] C. Danilowicz et al., PNAS 106, 13196 (2009).

[15] F. Küeher et al., Biophys. J. 92, 2491 (2007).

[16] S. Cocco,R.Monasson and JF.Marko, PNAS 98, 8608 (2001).

[17] J. F. Marko and E. D. Sigga, Macromolecules 28, 8759 (1995).

[18] M. Rief, H. C. Schaumann and H. E. Gaub, Nat. Struc. Bio. 6, 346 (1999).

[19] S. B. Smith, Y. Cui and C. Bustamante, Science 271, 795 (1996).

[20] J. Morfill et al., Biophys. J. 93, 2400 (2007).

[21] J. V. Mameren et al., PNAS 106, 18231 (2009).

[22] A. Lebrun and R. Lavery, Nucleic Acids Res. 24, 2260
(1996).

[23] R. A. Nehar and U. Gerland, Phys. Rev. Lett. 93, 198102 (2004).

[24] P. G. de Gennes, C. R. Acad. Sci. -Series IV-Physics 2, 1505 (2001).

[25] B. Chakrabarti and D. Nelson, J. Phys. Chem. B 113, 3831 (2009).

[26] M. P. Allen and D. J. Tildesley, Computer Simulations of Liquids (Oxford Science, Oxford, UK, 1987).

[27] D. Frenkel and B. Smit Understanding Molecular Simulation (Academic Press UK, 2002).

[28] N. Go and H. Abe, Biopolymers 20, 991 (1981); G. Mishra et al. J. Chem. Phys. 135, 035102 (2011).

[29] M. Kouza et al., Biophys. J. 89, 3353 (2005).

[30] M. S. Li, Biophys. J. 93, 2644 (2007).

[31] T. Hugel et al., Phys. Rev. Lett. 94, 48301 (2005)

[32] M. Santosh and P. K Maiti, J. Phys.: Cond. Matt. 21, 034113 (2009).

[33] J. Schluttig, M. Bachmann and W. Janke, J. Comput. Chem. 29, 2603 (2008).

[34] P. G. de Gennes, Scaling Concepts in Polymer Physics (Cornell Univ. Press, Ithaca, 1979).

[35] I. Rouzina and V. A. Bloomfield, Biophys. J. 80, 882 (2001).

[36] A. R. Singh, D. Giri and S. Kumar, J. Chem. Phys. 132, $235105(2010)$.

[37] J. Santalucia Jr., H. T. Allawi and P. A. Seneviratne, Biochemistry 35, 3555 (1996).

[38] W. Saenger, Principles of Nucleic Acid Structure (Springer-Verlag, Berlin, 1984).

[39] E. T. Kool, Annu. Rev. Biophys. Biomol. Struct. 30, 1 (2001). 